\title{
Del modelo didáctico práctico al modelo basado en el principio de investigación. Aplicación a la asignatura Typologies of Tourism Areas del grado de Turismo
}

Belén Pedregal Mateos

Universidad de Sevilla

Facultad de Geografía e Historia

Departamento de Geografía Humana

bpedregal@us.es

D.0.1.: http://dx.doi.org/10.12795/JDU.2018.i01.28

Pp.: $500-516$

\section{Resumen}

El Programa de Formación e Innovación Docente del Profesorado de la Universidad de Sevilla (FIDOP) pretende una estrategia de mejora docente gradual y progresiva basada en ciclos de mejora docente. En este contexto, se describe la experiencia de diseño y aplicación práctica de un ciclo de mejora en la asignatura impartida en inglés: Typologies of Tourism Areas del Grado Turismo. La mejora consistió fundamentalmente en el diseño e implementación de un modelo didáctico basado en el principio de investigación, aplicado a la impartición de un tema de la asignatura. Como resultado, cabe destacar que los estudiantes percibieron un avance en sus niveles de aprendizaje superior al evaluado mediante cuestionario, siendo en cualquier caso, positivo en su conjunto. 
Palabras clave: Tipología de espacios turísticos; Grado en Turismo; Docencia en lengua inglesa; Experimentación docente universitaria, Modelos Didácticos.

\section{Descripción del contexto de la intervención: docencia en lengua inglesa en el grado de Turismo}

La oferta de asignaturas impartidas en inglés en la Universidad de Sevilla no es muy elevada. En los últimos años, la Facultad de Turismo y Finanzas ha hecho una apuesta firme por reforzar esta oferta, mediante la promoción de grupos pequeños (máximo 30 plazas) y la asignación de aulas con bancos móviles en las que es posible fomentar el trabajo en grupo y la interacción entre los estudiantes. En esta comunicación se presenta la experiencia de la aplicación de un ciclo de mejora docente en una de las ocho asignaturas ofertadas en el Grado de Turismo en lengua inglesa, en concreto en la asignatura Typologies of Tourism Areas. Se trata de una asignatura obligatoria de 6 créditos de primer curso, para los estudiantes del grado de Turismo, en la que, por su condición de docencia en inglés, se matriculan otros estudiantes Erasmus de distintas nacionalidades, niveles y titulaciones que pueden estar relacionadas o no con los estudios de Turismo (por ejemplo Finanzas, Filología o Ciencias de la Educación, entre otras).

La diversidad de origen de los estudiantes junto con su condición multidisciplinar enriquecen el contexto educativo, mientras que el desigual nivel de madurez (estudiantes de cursos más avanzados junto con estudiantes de primer curso universitario) supone, en principio, una dificultad para el diseño de actividades que respondan a distintos niveles de complejidad y el desempeño de procesos educativos integradores.

La asignatura se imparte durante 15 semanas organizadas en 4 horas semanales, en sesiones de 2 horas cada una. Las sesiones que han sido objeto del ciclo de mejora 
fueron las correspondientes a la impartición del último tema de la asignatura, aunque el proyecto objeto de evaluación también comprendía los contenidos del tema anterior.

\section{Diseño del Ciclo de Mejora: repensando los contenidos, modelos metodológicos, actividades y evaluación}

El Programa de Formación e Innovación Docente del Profesorado de la Universidad de Sevilla (FIDOP) persigue una mejora docente gradual y progresiva basada en ciclos de mejora docente. La característica básica de estos ciclos es que con ellos se pretende mejorar el conjunto de la docencia, interviniendo en todas las variables fundamentales y principios básicos del entorno de aprendizaje (Bain, 2007), es decir, interviniendo en los contenidos, metodología, actividades y evaluación de las asignaturas, entendidas como variables interdependientes que se retroalimentan entre sí (Martín del Pozo et al., 2017). De esta forma, siguiendo a estos autores existen cuatro principios fundamentales articuladores de un modelo de enseñanza alternativo al tradicional transmisivo: 1) Tomar en consideración los modelos mentales de los que parten los estudiantes; 2) Formular los contenidos con preguntas clave; 3) Utilizar una metodología basada en la investigación y 4) Concebir la evaluación como un proceso que retroalimente el aprendizaje (ib., 2017, 25).

Teniendo en cuenta estos principios, en este apartado se resume el diseño de un ciclo de mejora docente dedicado a la impartición de un tema sobre las tendencias en el sector turístico. En él se tratan las nuevas tendencias desde el punto de vista de la oferta y la demanda, así como su impacto sobre nuevos modelos de implantación en destinos turísticos. Se presentan en primer lugar los contenidos vertebradores del tema, así como sus relaciones, para continuar con el modelo metodológico diseñado 
para su impartición, incluyendo las preguntas clave de partida y la secuencia de actividades diseñadas.

\section{Mapa de contenidos en red y preguntas-clave}

Constituye un error común programar una asignatura seleccionando únicamente contenidos conceptuales que normalmente son organizados en temas sin relación o jerarquía entre ellos (García Díaz et al., 2017). Por ese motivo el diseño del ciclo de mejora comienza con la reflexión sobre la propia metodología docente junto con la selección de los contenidos a enseñar y cómo organizarlos, conectarlos y problematizarlos. Este ejercicio se realiza mediante la elaboración de un "mapa de contenidos", en el que se representa de manera jerarquizada y relacionada "todo aquello que queremos que los estudiantes aprendan" (ib. 56), incluyendo conceptos, procedimientos y actitudes.

En el caso del tema dedicado al análisis de las previsiones turísticas (ver figura 1) se han seleccionado dos grandes bloques conceptuales vertebradores del tema: 1) Previsiones desde el lado de demanda y 2) Desde el lado de la oferta. A partir de estos dos grandes bloques se seleccionan los principales conceptos (enmarcados en figuras cuadradas sombreadas): 1.1. Las previsiones de población; 1.2. Los mercados emergentes; y 1.3. Las tendencias sociotecnológicas y políticas, para el lado de la demanda y 2.1. El turismo experiencial y 2.2. Los espacios corporativos de ocio, como nuevas tendencias del lado de la oferta. De éstos, a su vez, parten nuevos conceptos clave (representados mediante cuadrados simples) entre los que podemos mencionar: el envejecimiento de la población, la emergencia de las clases medias chinas e indias, la generalización del tiempo de ocio en las sociedades desarrolladas, entre otros factores claves a tener en cuenta en los nuevos escenarios socioeconómicos. Las relaciones entre sí, se representan mediante línea azul, para los contenidos conceptuales, y mediante línea roja en el caso de 
los contenidos actitudinales (attitudinal skills) y procedimentales (instrumental skills) que se trabajan mediante las actividades que más adelante se detallan. Entre ellas cabe señalar la elaboración de infografías a integrar en las presentaciones orales de los trabajos finales-como contenidos instrumentales- o la actitud critica y conciencia medioambiental -como contenidos actitudinales relacionados con el concepto de turismo responsable-.

Una vez elaborado el mapa de contenidos, se procede

$$
\text { Topic } 5 \text { Contents: New Trends in Tourism }
$$

Attitudinal skills

Instrumental skills

Socioeconomic trends and their effect on the demand-side

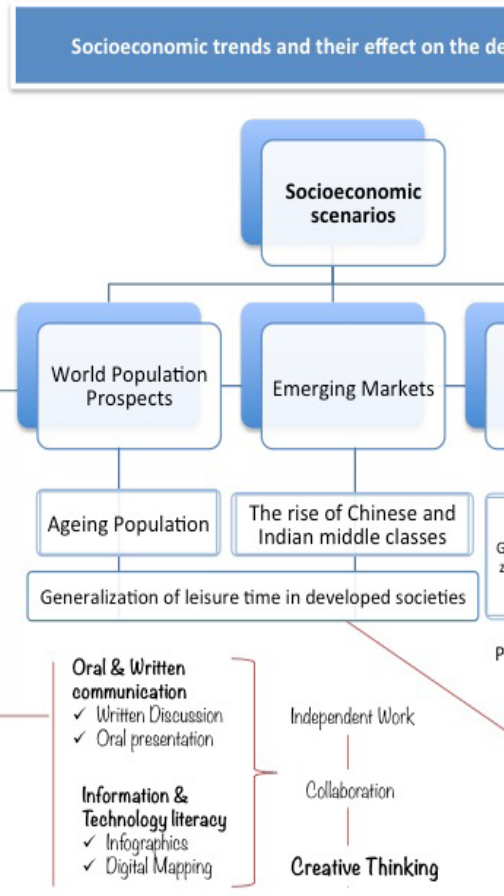

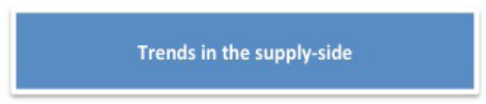

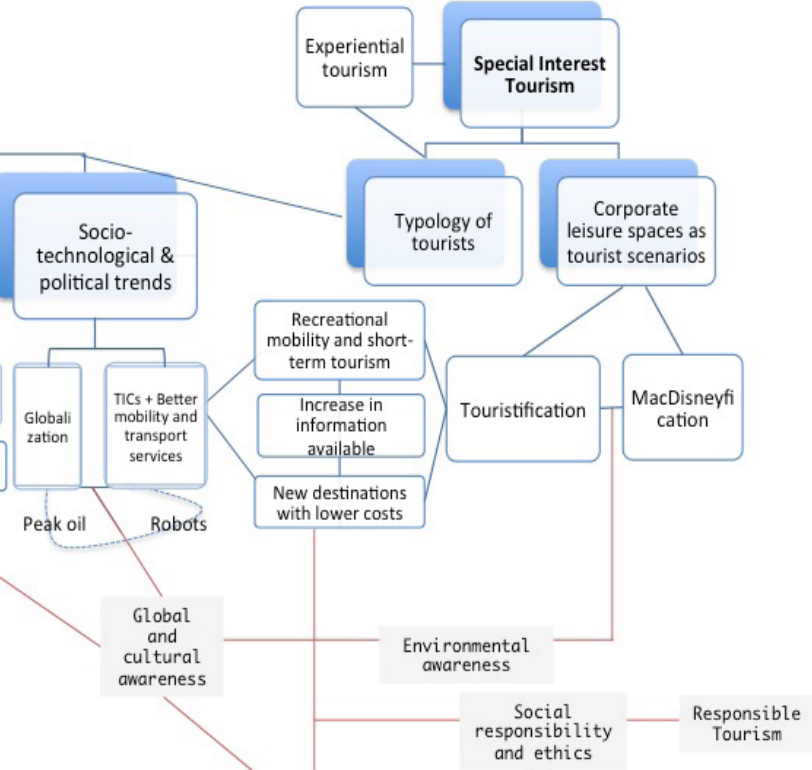

Critical thinking

Figura 1. Mapa conceptual del tema New Trends in Tourism

a la "problematización" del tema mediante el diseño de las actividades por sesión y la formulación de preguntas clave iniciales que posteriormente se responderán con las actividades diseñadas para tal fin. En este caso se formularon tres preguntas sobre "el turista del futuro" con el objetivo 
de estimular la creatividad e imaginación de los estudiantes. En concreto las preguntas iniciales fueron: 1) How do you imagine the tourist profile/s for the late 21st century?; 2) What aspects from the demand-side should we consider in order to make projections for the tourism industry?; 3What do you think the tourist will be doing in the future? Refer to duration of vacation; activities \& destinations. En el siguiente apartado se presentan las actividades diseñadas para dar respuesta a estas preguntas.

\section{Modelo metodológico, secuencia de actividades y cuestionario inicial-final.}

A grandes rasgos, los estudios sobre estilos docentes universitarios identifican dos grandes modelos generales de docencia: uno "centrado en el profesor y los contenidos", donde el foco se pone en los conocimientos disciplinares del propio docente; y otro centrado "en el estudiante y su aprendizaje", en el que la docente orienta al estudiante a construir el conocimiento y a cambiar sus visiones sobre lo que está estudiando (Martín del Pozo, et al., 2017). En un paso más hacia la concreción de los modelos sobre la metodología de la enseñanza, De Alba y Porlán (2017) distinguen tres grandes grupos según estén basados en: 1) la transmisión; 2) la teoría y su aplicación práctica o 3) en la reelaboración de ideas de los estudiantes. Sobre este último añaden su propia propuesta de modelo basado en el "aprendizaje por investigación" (ib. 2017, 45).

La implementación del ciclo de mejora docente ha consistido en analizar nuestro modelo metodológico habitual para diseñar y aplicar un modelo más complejo basado en el principio de investigación y reelaboración de ideas por parte de los estudiantes. En síntesis este modelo parte siempre de una cuestión, que puede estar formulada como problemas, preguntas, proyectos, etc., que ha de activar el interés, implicación y respuestas del estudiante. Estas respuestas han de ser confrontadas con 
nuevas informaciones (diversidad de recursos y actividades), así como con ideas o experiencias previas que se reactivan, para acabar concluyendo y comparando las ideas iniciales y finales. Desde el punto de vista epistemológico, se asume que el conocimiento es siempre una construcción relativa, susceptible de ser reelaborada y reformulada (ib. 2017,41). En esta asignatura, la metodología de enseñanza habitual consistía en trabajar mediante proyectos relacionados con las salidas profesionales (Aprendizaje basado en Proyectos), encuadrándose la metodología de enseñanza habitual en el modelo "basado en la teoría y su aplicación práctica”. Para evolucionar a un modelo más complejo, propuesto por los formadores, se diseñó el modelo metodológico representado en la figura 2, que comienza con el planteamiento inicial de unas preguntas clave de formulación abierta, cuyas respuestas se elaboran individualmente y posteriormente se ponen en común. Seguidamente se realizan una serie de actividades de contraste que se detallan más adelante, para finalizar con el replanteamiento de las cuestiones iniciales. En concreto, se ha diseñado un cuestionario de tres preguntas abiertas que los estudiantes responden por escrito en la primera sesión antes de comenzar el tema. Al finalizarlo, se les devuelve con la instrucción de reaccionar a las primeras respuestas: comentando o añadiendo nuevos contenidos (en color distinto al texto inicial).

Jornadas de Formación e Innovación Docente del Profesorado | № 1 (2018) Esta obra se distribuye con la licencia Creative Commons 


\section{Preguntas iniciales}

Elaboración de conclusiones sobre ideas finales e iniciales
Respuestas individuales de

los estudiantes y puesta en

común

\section{Actividades de contraste}

- Explicaciones, Lecturas

- Visionado/Comentario de

Videos

- Elaboración de Proyectos

Supervisados

Figura 2. Modelo metodológico diseñado para la asignatura Typologies of Tourism Areas (adaptado de De Alba y Porlán, 2017, 41).

Las actividades de contraste se detallan a continuación por sesión y en relación a las cuestiones iniciales:

Preguntas claves 1 y 2: How do you imagine the tourist profile/s for the late 21st century?; What aspects from the demand-side should we consider in order to make projections for the tourism industry?

Estas preguntas se responden con el bloque 1 del mapa de contenidos denominado "Socioeconomic trends and their effect on the demand-side". Para tratar estos contenidos se han diseñado las siguientes actividades:

- Sesion 1: Visionado y puesta en común en clase del video: "Don't Panic, the world might not be as bad as you believe" sobre proyecciones demográficas y socieconómicas mundiales, para comentar el efecto que tendrán en el sector turístico el envejecimiento de la población mundial y el crecimiento de 
la población, con particular atención al crecimiento de las clases medias chinas e indias.

- Sesión 2- 1 hora: Presentación por parte de la profesora de las previsiones económicas de la OCDE, algunas noticias sobre política internacional y un articulo que deben leer sobre factores que afectan la demanda turística en el presente y futuro.

Sesión 2- 1 hora: Trabajo en grupo supervisado: Teniendo en cuenta estos contenidos los estudiantes deben hacer un análisis DAFO (Debilidades-Amenazas-Fortalezas-Oportunidades) de una ciudad de su elección como destino turístico que habrán de presentar en las últimas semanas de clase. Este proyecto fue propuesto en el tema anterior dedicado al turismo urbano y lo desarrollan teniendo en cuenta los contenidos de estos dos últimos temas del temario de la asignatura. El objetivo instrumental que se persigue es que los estudiantes conozcan cómo se elabora un plan estratégico urbano, cómo se realiza el diagnóstico y cómo se proponen objetivos y acciones de acuerdo al diagnostico realizado mediante la técnica DAFO, aplicada a una ciudad concreta de su elección. El diagnostico deben realizarlo conforme a los contenidos tratados en el tema 4 (turismo urbano) y las acciones y objetivos turísticos que deben proponer tienen que basarse en las previsiones del sector (tema 5 objeto del ciclo de mejora) y estar alineados con el principio de sostenibilidad y responsabilidad turística, trabajados a lo largo del curso.

Pregunta 3: What do you think the tourist will be doing in the future? Refer to duration of vacation; activities \& destination.

Esta pregunta se responde con el bloque 2 del mapa de contenidos: "Trends in the supply-side". Para tratar estos contenidos se diseñaron las siguientes actividades: 
- Sesión 3- 1 hora: Presentación por parte de la profesora del concepto Special Interest Tourism y las nuevas tendencias en las ofertas turísticas. Esta presentación es compartida en enseñanza virtual.

- Sesión 3- 1 hora: Trabajo en grupo supervisado. Teniendo en cuenta todos los materiales, presentaciones y comentarios realizados en clase para los dos últimos temas (tema 4 turismo urbano y tema 5 nuevas tendencias turísticas) los estudiantes deben proponer acciones que contribuyan a alcanzar el turismo urbano sostenible en una ciudad concreta de su elección. Estos contenidos se enmarcan en un plan estratégico urbano. Los resultados se presentan en clase durante las ultimas sesiones del cuatrimestre.

- Sesiones 4 a 7: Presentación de los proyectos de los estudiantes en grupos de dos. En la última sesión reciben sus respuestas iniciales por escrito y se les pide que contesten de nuevo reaccionando, añadiendo y/o comentando sus primeras respuestas (utilizando un color distinto para la escritura). Los resultados se presentan en el siguiente apartado.

\section{Del diseño a la práctica: Aplicación del ciclo de mejora y evaluación del aprendizaje de los estudiantes}

\section{Relato resumido de las sesiones}

A lo largo del cuatrimestre los estudiantes de esta asignatura han experimentado con numerosos recursos y actividades de aprendizaje diversas. Por ese motivo, el inicio del tema por medio de un cuestionario de preguntas abiertas en las que se les pedía fueran creativos no les resultó una práctica fuera de lo común, a pesar de ser la 
primera vez que se implementó en clase. Las preguntas pretendian fomentar su interés y desplegar un ambiente positivo, sin embargo, algunos estudiantes demostraron poca motivación quizás por tratarse de una prueba en la que tienen que realizar un esfuerzo para expresarse en inglés por escrito.

En esa primera sesión se les indicó que estos cuestionarios se les devolverían al finalizar el tema para que confrontaran sus ideas iniciales y finales, sin embargo, al final muy pocos recordaron que se les pediría responder de nuevo, lo que les causó cierta reticencia inicial. Quizás el hecho de que este ejercicio no puntuara motivó al principio cierta despreocupación por su parte, unido al hecho de que el cuestionario se les devolvió el último día de clase, después de varias sesiones de presentación de proyectos, lo que constituyo probablemente un periodo de tiempo excesivo.

De las actividades programadas en este tema el visionado y puesta en común del video sobre previsiones de la población mundial fue la que suscitó el mayor interés y participación por parte de los estudiantes. En contraste, las horas de trabajo en grupo resultaron poco provechosas. En estas horas se distribuyeron en el aula según los grupos de trabajo, para que plantearan sus dudas y avances sobre los proyectos en marcha. Mientras un grupo es supervisado, el resto debe trabajar y avanzar en sus proyectos, no obstante en estas sesiones varios grupos demostraron poca predisposición al trabajo en aula. Todos los grupos son supervisados individualmente, comprobándose que la mayoría ha avanzado muy poco en el trabajo final y solo algunos grupos muestran sus dudas y resultados preliminares.

En las sesiones dedicadas a la presentación de los proyectos finales de la asignatura la mayoría mostraron interés por las presentaciones de sus compañeros, probablemente porque van seguidas de un breve comentario y retroalimentación por mi parte, haciendo referencia a las 
debilidades y fortalezas en la adquisición de los conocimientos en relación a los criterios de valoración de los trabajos que son compartidos en la plataforma de enseñanza virtual.

\section{Evaluación del aprendizaje de los estudiantes}

Por lo que se refiere a la evaluación del aprendizaje, en este ciclo de mejora se aplicaron tres instrumentos: 1) cuestionario de tres preguntas claves abiertas (inicial-final); 2) Autoevaluación del estudiante -ambos sin repercusión en la calificación final-y 3) Calificación del proyecto elaborado en grupos de dos y presentado en clase. Este proyecto les había sido propuesto antes de la aplicación del ciclo de mejora: el análisis DAFO de una ciudad como destino turístico y la propuesta de acciones para contribuir a la consecución de un turismo sostenible urbano. Los contenidos del tema objeto del ciclo de mejora debían aplicarlos en la propuesta de acciones y objetivos estratégicos de la ciudad respecto a la consecución de un turismo urbano responsable y sostenible.

Los resultados del cuestionario se presentan mediante la escalera de aprendizaje propuesta por Rivero y Porlán (2017) en la que se muestra conjuntamente los niveles iniciales y finales de los estudiantes (figura 3). Si bien es cierto que los resultados finales muestran una mejora general respecto a algunos contenidos clave del tema, es de destacar que casi la mitad de la clase (el 45\%) se queda en los dos niveles más bajos de progreso y sólo el $15 \%$, es decir, 3 de los 20 estudiantes que responden a ambos cuestionarios, demuestran una clara comprensión de los aspectos que determinan las tendencias futuras en la demanda y oferta turísticas: el resto ha avanzado fundamentalmente en la identificación de los aspectos que determinan el perfil turístico. Estos resultados son acordes con las calificaciones obtenidas por los proyectos elaborados para estos dos temas, pues 16 de los 26 estudiantes 
evaluados obtuvieron buenos resultados -un 61\%- mientras que el 39\% restante suspendió el trabajo. Este hecho puede relacionarse con la dificultad de algunos estudiantes a la hora de trabajar con textos escritos y especialmente, en inglés, ya que los contenidos relacionados con los niveles superiores de la escalera se trabajaron a partir de la lectura de un articulo, mediante preguntas a responder en grupo y puesta en común en clase.

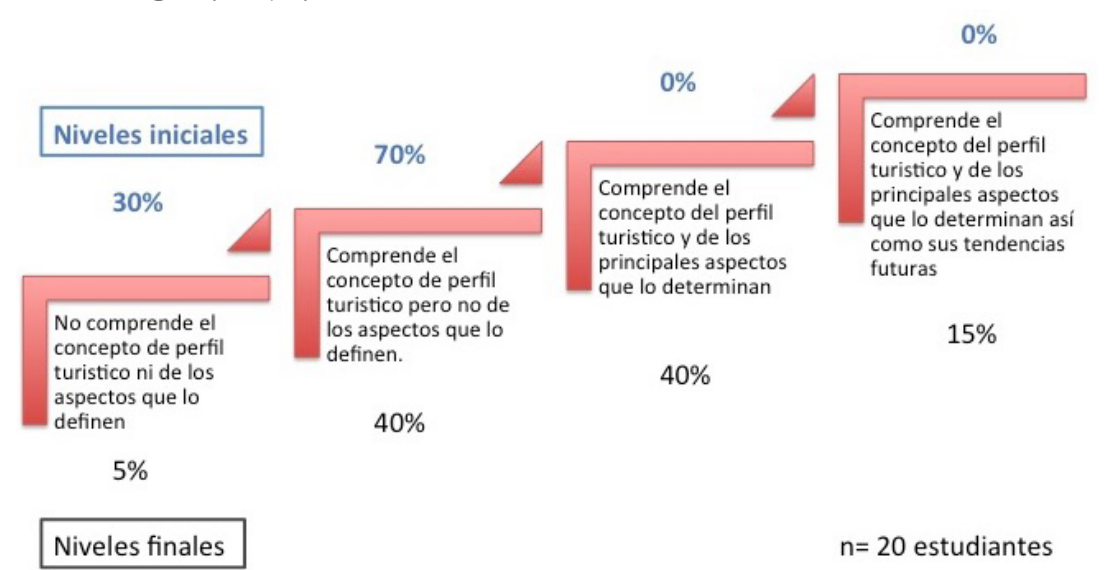

Figura 3. Escalera de aprendizaje inicial y final del tema New Trends in tourism.

Por lo que respecta al segundo instrumento de evaluación aplicado durante el ciclo de mejora, en la última sesión de clase se les pidió que expresaran anónimamente por escrito la valoración de su aprendizaje durante el tema. Como resultado, 17 de los 23 estudiantes presentes (un 74\%) valoraron muy positivamente la adquisición de conocimientos en este tema, mientras que 6 (26\%) manifestaron no haber aprendido, de lo que se deduce la valoración mayormente positiva por parte de los estudiantes de su propio rendimiento y de la metodología aplicada. 


\section{Reflexiones personales finales: evaluación del ciclo de mejora}

Durante el curso de formación y aplicación del ciclo de mejora, hemos tenido la oportunidad de iniciarnos en el diseño de experiencias (actividades, problemas) que ayudan a los estudiantes a aprender, siguiendo la propuesta de Finkel (2000). Según este autor la característica fundamental a tener en cuenta es que estas actividades (preguntas o problemas) deben estimular la curiosidad y el razonamiento, como ocurre con los enigmas. Además, se deben añadir otras instrucciones o conjunto de actividades que consigan un "aprendizaje sostenido" de tal forma que sirvan de guía al estudiante en su proceso de aprendizaje.

En este ciclo, implementé el planteamiento de cuestiones clave sobre una metodología de aprendizaje basada en proyecto ya en marcha, las preguntas se plantearon sobre una parte de los contenidos del proyecto, no de todo el proyecto, por lo que la introducción de distintas actividades con distintos enfoques sobre los mismos contenidos resultó un poco confusa para gran parte de la clase, especialmente para los estudiantes más jóvenes del grupo. Por ese motivo, los resultados objetivos obtenidos en la evaluación del aprendizaje no fueron tan positivos como me esperaba. A ello hay que unir el hecho de que las preguntas claves se diseñaron como instrumento de estimulo y evaluación del aprendizaje individual, no para trabajarlas explícitamente en grupo, ya que tenían asignados proyectos grupales con anterioridad, por lo que la superposición de ambas actividades resultó probablemente excesiva y no se complementaron claramente hacia la consecución de los objetivos perseguidos.

Durante la aplicación del ciclo de mejora mi metodología docente varió respecto a la introducción explícita de "actividades de investigación" mediante la definición y propuesta de preguntas claves, estimulantes. Con anterioridad solía plantear la realización de proyectos relacionados con 
la materia y salidas profesionales, es decir, había fomentado un enfoque práctico pero que no había sido formulado explícitamente a través de una metodología docente basada en el principio de investigación.

Tengo que reconocer que la problematización de los temas, formulación de cuestiones claves y sobre todo, el análisis de los resultados encierran gran dificultad, pues es fácil formular preguntas que generen respuestas de informaciones o contenidos, pero es más complicado formular preguntas o problemas que sean capaces no sólo de despertar el interés de los estudiantes, sino también de orientarlos hacia procesos de aprendizaje más complejos consistentes en "alguna actividad intelectual de orden superior" (Bain, 2007) es decir, que los anime a comparar, aplicar, evaluar, analizar y sintetizar, no sólo a escuchar y recordar.

Además, de sus respuestas, hemos inferido modelos o esquemas de pensamiento inicial y final para evaluar su progreso, lo que también entraña cierto grado de dificultad si no se está entrenada para ello. Especialmente complicado me ha resultado establecer los grupos de ideas y respuestas iniciales así como ordenarlas según su grado de elaboración y complejidad. Hasta el momento no había pensado en los contenidos de las asignaturas según estos parámetros, pues me había centrado más en transmitir correctamente contenidos y evaluar el grado de adquisición de los mismos que en conocer y comprender los modelos mentales de los que parten los estudiantes, sus procesos de cambio y los obstáculos que dificultan su progresión. Sin embargo creo que esta cuestión es fundamental, sobre todo para el caso de grupos de estudiantes heterogéneos como éste: con diferentes niveles de madurez, distinta formación y distinto conocimiento del inglés -el idioma empleado-, por lo que a partir de ahora intentaré diseñar actividades que me ayuden a plantear la evaluación en estos términos. 
En conclusión, durante la realización del curso y aplicación del ciclo de mejora he aprendido que hasta ahora aplicaba un modelo metodológico basado fundamentalmente en la teoría y su aplicación práctica (De Alba y Porlán, 2017), con la variante de situar en muchos casos la práctica antes de la teoría e inferir ésta en sesiones participativas de conclusiones y presentación de contenidos por mi parte. Durante la aplicación del curso de mejora he constatado que los instrumentos o técnicas aplicadas en clase no son tan importantes como los principios básicos creadores del mejor entorno posible para el aprendizaje, entre ellos la aplicación del principio de investigación. Hasta el momento no me había planteado el diseño de enigmas o la resolución de problemas que estimulen tan directamente la indagación, el contraste y construcción de conocimiento por parte de los estudiantes. Creo, sin embargo, que es una buena técnica para estimular la motivación, la colaboración y el trabajo en grupo, por lo que intentaré este planteamiento en sucesivos ciclos de mejora.

Finalmente, tampoco me había planteado hasta ahora enseñar a los estudiantes a problematizar los fenómenos y creo que en sucesivos cursos constituirá uno de los grandes retos que tengo por delante, junto con la aplicación de la evaluación de los esquemas y modelos de pensamiento iniciales y finales.

Jornadas de Formación e Innovación Docente del Profesorado | № 1 (2018) Esta obra se distribuye con la licencia Creative Commons 


\section{Referencias bibliográficas}

Bain, K. (2004). Lo que hacen los mejores profesores universitarios. Valencia: Publicaciones Universidad de Valencia.

De Alba, N., Porlán, R. (2017). La metodología de la enseñanza. En Porlán, R. (coord.) Enseñanza Universitaria, Cómo mejorarla (pp. 37-53). Madrid: Editorial Morata.

Finkel, D. (2000): Experiencias que enseñan: Crear esquemas para el aprendizaje. En Dar clase con la boca cerrada (pp. 153-183), Valencia: Universitat de València.

García Díaz, E., Porlán, R. y Navarro, E. (2017). Los fines y los contenidos de la enseñanza. En Porlán, R. (coord.) Enseñanza Universitaria, Cómo mejorarla (pp. 23-36). Madrid: Editorial Morata.

Martín del Pozo, R., Pineda, J.A., y Duarte, O. (2017). La formación docente del profesorado universitario. En Porlán, R. (coord.) Enseñanza Universitaria, Cómo mejorarla (pp. 55-72). Madrid: Editorial Morata.

Porlann, R. (2017). Enseñanza Universitaria, Cómo mejorarla. Madrid: Editorial Morata.

Rivero y Porlán, (2017): La evaluación en la enseñanza universitaria. En Porlán, R. (coord.) Enseñanza Universitaria, Cómo mejorarla (pp. 73-91). Madrid: Editorial Morata.

Jornadas de Formación e Innovación Docente del Profesorado | № 1 (2018) Esta obra se distribuye con la licencia Creative Commons 